\title{
Evolución histórica de las repoblaciones forestales en la Comunitat Valenciana y su relación con los incendios forestales
}

\author{
Sancho Llansola J. ${ }^{1 *}$, Pons Ruiz A. ${ }^{2}$, Escrig del Valle A. ${ }^{3}$ \\ ${ }^{1}$ VAERSA. C/Alcalde Cano Coloma 4. 46022-VALENCIA (España). \\ ${ }^{2}$ Ejercicio libre de la profesión. C/ en Sanz 12-8 46001-VALENCIA (España). \\ ${ }^{3}$ PYRO FIRE EXTINCTION SL. Centro de Desarrollo Empresarial - Edificio 9B. Universitat Politècnica de València. \\ Camino de Vera s/n - 46022 VALENCIA (España). \\ * e-mail: jsancho@vaersa.org
}

\section{Resumen}

El paisaje de la Comunitat Valenciana ha sido modulado en gran medida por las cerca de 190000 hectáreas que han sido objeto de repoblación forestal, principalmente a lo largo del siglo XX y tradicionalmente con objetivos protectores. Estas actuaciones forestales, en muchos casos sin un mantenimiento y gestión posterior, han derivado en masas que habiendo cumplido en cierto grado con la potenciación de los servicios ambientales de regulación hidrológica y conservación de suelos, han provocado en algunos casos la minoración de otros, principalmente relacionados con la estabilidad de las masas y los incendios forestales. En el presente trabajo se estudia, por una parte, la distribución por provincias de los trabajos de repoblación y su evolución en el tiempo, y por otra, se analizan las áreas que tras haber sido repobladas han sufrido del paso de un incendio. Las fuentes de información básicas empleadas son la cartografía disponible de áreas repobladas desde 1883, elaborada por la Conselleria d'Infraestructures Territori i Medi Ambient; la cartografía de perímetros de incendios, facilitada por la Conselleria de Governació i Justícia, disponible desde 1978 para la provincia de Valencia y desde 1993 para las de Castellón y Alicante, así como la cartografía del Inventario Forestal Nacional y la del Plan de Acción Territorial Forestal de la Comunitat Valenciana aprobado en 2013. Considerando la información que proporciona del estado de la vegetación el Tercer Inventario Forestal Nacional se obtiene una aproximación de la efectividad del esfuerzo repoblador. La destrucción de la cubierta vegetal que provocan los incendios puede incidir en el aumento de la pérdida de suelo por erosión y en el equilibrio hidrológico, por lo que los resultados podrán servir de apoyo en la toma de decisiones futuras, en la planificación de nuevas inversiones y en el diseño de las actuaciones de restauración forestal que lleve a cabo la administración.

Palabras clave: Diseño de repoblaciones, inventario forestal nacional, política forestal, prevención de incendios forestales, regeneración natural, superficie arbolada. 


\section{Introducción}

Según Serrada (2000), las repoblaciones forestales engloban aquellas técnicas necesarias para crear una masa forestal estable, formada por especies vegetales leñosas, en un terreno cuya vegetación actual es ineficaz en mayor o menor grado según el uso asignado al territorio, y que adoptando las características deseadas, cumpla los fines que de ella se demanden. Por otro lado, se define como incendio forestal al fuego que se extiende sin control sobre terreno forestal, afectando a vegetación que no estaba destinada a arder (Vélez, 2000).

De esta forma, se puede afirmar de forma simplificada que las repoblaciones y los incendios forestales son procesos opuestos, ya que mientras que en el primero, generalmente se incrementa la carga de material vegetal, en el segundo se elimina; mientras que con las repoblaciones se busca formaciones más maduras, los incendios originan matorrales con menor grado de evolución. Las repoblaciones buscan, entre otros objetivos, la regulación de los procesos hídricos y la conservación de suelo, sin embargo, los episodios de fuego pueden provocar importantes procesos de escorrentía y erosión debido a que a menor cobertura vegetal mayores son las pérdidas de suelo (Mac Donald and Robichaud, 2008).

La importancia de ambos radica en que han sido dos de los agentes que más han moldeado el paisaje forestal autonómico, ya que únicamente considerando que desde finales del siglo XIX cerca de 190000 hectáreas fueron repobladas y que los incendios, teniendo en cuenta sólo el último tramo del siglo XX y primeros del XXI, han afectado a más de 345000 ha (en una o varias ocasiones), se puede decir que la cuarta parte de la superficie forestal autonómica que ocupa 1,3 millones de hectáreas (PATFOR, 2013) ha sufrido al menos un incendio.

La falta de gestión de las grandes repoblaciones llevadas a cabo a mitad de siglo XX, ha sido origen de grandes y continuas formaciones monoespecíficas de pinar que han sufrido el efecto de los incendios forestales, con gran capacidad de propagación en estas zonas por la inexistencia de un paisaje en mosaico, la elevada carga de combustible, la falta de puntos de control y la homogeneidad reinante. Una vez afectadas por el fuego, en estas zonas, dependiendo de la capacidad de regeneración del bosque existente, se han llegado a alcanzar densidades de regeneración natural superiores a 100000 pies ha $^{-1}$ (De las Heras et al., 2011), lo que, ante la generalizada falta de gestión de estas masas, derivaba en la presencia de nuevas masas con una gran susceptibilidad a arder fuera de capacidad de extinción (Costa et al., 2011).

Las zonas incendiadas, han sido objeto prioritario de las restauraciones realizadas en el ámbito autonómico principalmente mediante la ejecución de repoblaciones protectoras (Pemán y Navarro, 1998), con el objetivo de minimizar las pérdidas de suelo. Estas actuaciones, en muchas ocasiones no se ejecutaban pensando en la posibilidad de un nuevo episodio de fuego y no iban acompañadas del desarrollo de medidas preventivas, así como tampoco de una posterior gestión de la masa, lo que facilita su nueva afección por un incendio y la consiguiente pérdida económica y ambiental. 


\section{Objetivos}

El objetivo principal es conocer la evolución y situación actual de las repoblaciones forestales en la Comunitat Valenciana, así como el efecto de los incendios forestales sobre dicha superficie. Para alcanzar este objetivo general, se han abordado a su vez los siguientes objetivos específicos:

- Describir la actividad repobladora desde finales del siglo XIX a principios del XXI.

- Cuantificar qué superficie repoblada ha llegado a ser arbolada.

- Conocer qué actuaciones de repoblación se han visto afectadas por el fuego.

- Conocer qué superficie repoblada ha sufrido un incendio posteriormente.

\section{Material y métodos}

La metodología seguida se ha basado fundamentalmente en cruzar mediante procedimientos de Sistemas de Información Geográfica, la cartografía descriptiva de las repoblaciones forestales, realizada por la Conselleria d'Infraestructures, Territori i Medi Ambient, la del Tercer Inventario Forestal Nacional para describir el estado actual de la vegetación y la cartografía de incendios forestales, desarrollada por la Conselleria de Governació i Justicia, describiéndose a continuación las principales características de cada una de ellas.

\subsection{Cartografía de repoblaciones}

La principal fuente de información respecto a la caracterización de las repoblaciones en la Comunitat Valenciana es un estudio realizado por el Servicio de Ordenación y Gestión Forestal. Este estudio se realizó con el objetivo general de caracterizar las repoblaciones forestales ejecutadas en el ámbito autonómico en un periodo de tiempo que abarca desde 1883 hasta 2014. Todavía quedan algunos proyectos por incorporar pero se estima que suponen unas 150 ha, superficie que no altera de forma significativa los análisis realizados. El estudio se realizó a partir de información procedente, principalmente, de expedientes de los proyectos de repoblación del archivo de la Conselleria d'Infraestructures Territori i Medi Ambient, de documentación de los consorcios de repoblación y de información facilitada por Servicio de Ordenación y Gestión Forestal, los Servicios Territoriales y los Agentes Medioambientales.

La información básica de partida para la preparación de la cartografía fue la memoria y los planos de localización de los trabajos de las repoblaciones de los proyectos ejecutados por el Estado o la Conselleria, excluyéndose del análisis las ejecutadas por particulares o por cualquier otra entidad pública.

La escala de trabajo fue muy distinta dependiendo de la fuente de información, en algunos casos se disponía únicamente de un croquis de localización de los rodales mientras que en otros de fecha más reciente se disponía directamente de la cartografía digital de los proyectos originales en formato vectorial. Tras la incorporación de los datos, se corregían a partir de fotointerpretación según la vegetación observa- 
da, o la preparación del suelo.

La cartografía generada refleja una superficie repoblada hasta 1996 de 149573 , muy cercana a las 158869 ha del estudio presentado por Reyna Doménech y Fernández Guijarro en el año 2000 el cuál se realizó en base a los resúmenes de actuaciones de las Secciones Forestales, presentando únicamente un 6\% de diferencia.

Existen zonas que han sido repobladas en más de una ocasión, distinguiéndose en el análisis cuando estas zonas se contaban por separado o una única vez. De igual forma, también hay que indicar que las reposiciones de marras, recogidas en un proyecto distinto al de la primera ejecución, se han considerado una repoblación diferente a la primera.

\subsection{Cartografía del Inventario Forestal Nacional III (IFN3)}

Hasta la fecha, se han realizado tres Inventarios Forestales Nacionales, 19661975, 1986-1996, 1997-2007, empleando éste último en el presente trabajo, para analizar la situación actual de las repoblaciones ejecutadas.

El Mapa Forestal de España a escala 1:50 000 (MFE50) en el que se apoya la elaboración del IFN3, fue elaborado en base a fotos aéreas de 1999, aunque se actualizó aprovechando los trabajos de campo del IFN3 en la Comunitat Valenciana en 2006. Habiendo otras cartografías disponibles más recientes y con una escala más detallada, como puede ser la ofrecida por el Sistema de Ocupación del Suelo en España (SIOSE), se ha considerado emplear la ofrecida por el IFN3 por su recurrencia decenal, por tener disponibles cartografías de años anteriores así como del resto de España, lo cual permite ampliar el área de estudio, así como porque centra su caracterización en las masas arboladas. Dentro de esta cartografía, se han considerado como superficies arboladas aquellas áreas en las que la clasificación de la vegetación del IFN3 se correspondía con monte arbolado, monte arbolado ralo, monte arbolado disperso o árboles fuera del monte.

\subsection{Cartografía de incendios}

La cartografía de incendios forestales, tal como se ha indicado, ha sido facilitada por la Conselleria de Governació i Justícia, que la elabora anualmente. La disposición temporal de esta cartografía varía de una provincia a otra, ya que está disponible desde 1978 en Valencia, mientras que en Castellón y Alicante los incendios más antiguos recogidos datan de 1993.

La forma de elaboración y su precisión, se ha ido modificando y mejorando en el tiempo, especialmente gracias a la generalización de la tecnología GPS y a la teledetección, con lo que los perímetros de los incendios de los últimos años están mucho mejor definidos que los primeros de la serie.

\subsection{Procesamiento de la información}

En base a la cartografía de repoblaciones, en primer lugar se realiza una cuanti- 
ficación superficial de éstas, tanto como por provincias como por decenios, obteniendo la importancia relativa de la superficie repoblada con la forestal existente, tomando como valor la ofrecida por el PATFOR (2013).

De igual forma también se analizan las superficies repobladas en función del momento histórico en el que se encuentran, dividiéndose en los siguientes períodos:

- 1883-1939: desde el momento de que se dispone de información cartográfica de las repoblaciones hasta el final de la guerra civil. Coincide con finales del siglo XIX, periodo en el que se dicta la primera legislación con vocación específicamente repobladora, como la Ley de 9 de junio de 1877 sobre Repoblaciones, Fomento y Mejora de los Montes Públicos y se crea el Servicio de Repoblaciones de Cabeceras de Cuencas (1888) y el Servicio Hidrológico Forestal (1901) (Pemán y Navarro, 1998).

- 1940-1975: años en los que se desarrolló principalmente el Plan Nacional de Repoblaciones, sustituto del Plan General de Repoblaciones de 1926, y en que se reorganizó el Patrimonio Forestal del Estado (1941) con un marcado carácter repoblador (Martínez, 1953).

- 1976-1984: periodo de transición política en el que se redujo el ritmo anual de repoblaciones en toda España (Pemán y Navarro, 1998).

- 1985-2014: desde la cesión de las competencias en materia forestal a las comunidades autónomas hasta la actualidad.

Aunque la finalidad de las repoblaciones puede ser diferente, la gran mayoría persigue crear masas arboladas como medio para la consecución de sus objetivos. De esta forma, se ha evaluado la efectividad de la actividad repobladora analizando qué porcentaje de las repoblaciones ejecutadas conforman actualmente este tipo de masas. Tal como se ha explicado, la cartografía que se ha empleado para conocer el estado actual de las masas ha sido la correspondiente al IFN3. Al mismo tiempo se considera que han de pasar 10 años para que una repoblación alcance 3 metros de altura y aparezca catalogada como arbolada en el IFN3, en base a la tabla de producción de Pinus halepensis de Montero (2000). Por lo tanto, para evaluar la efectividad de las repoblaciones únicamente se ha trabajado con las realizadas hasta 1996.

Por último, a partir de la cartografía de incendios facilitada por la Conselleria de Governació i Justícia, se han cruzado los datos para conocer qué superficie de la que había sido repoblada ha sufrido posteriormente un incendio y que fracción de ésta se considera arbolada en el IFN3.

\section{Resultados y discusión}

\subsection{Superficie repoblada por provincias}

Dentro del periodo analizado (1883-2014) en la Comunitat Valenciana se han repoblado 185098 ha, concentrándose más de la mitad en la provincia de Valencia, con cerca de 100000 ha, situándose posteriormente Alicante con más de 50000 y Castellón con menos de 40000 ha (Tabla 1). 
Tabla 1. Superficie forestal y superficie repoblada por provincia en el periodo 1883-2014, diferenciando las zonas que se han repoblado en más de una ocasión (superposición).

\begin{tabular}{|c|c|c|c|c|c|}
\hline \multirow[b]{2}{*}{ Provincia } & \multicolumn{3}{|c|}{ Superficie repoblada (ha) } & \multirow{2}{*}{$\begin{array}{c}\text { Superficie } \\
\text { forestal (ha) } \\
(\text { PATFOR, 2013) }\end{array}$} & \multirow{2}{*}{$\begin{array}{l}\% \text { de superficie } \\
\text { forestal sobre } \\
\text { la que se han } \\
\text { realizado } \\
\text { repoblaciones }\end{array}$} \\
\hline & Total & Superposiciones & $\begin{array}{c}\text { Descontando } \\
\text { superposiciones }\end{array}$ & & \\
\hline Castellón & 35714 & 3384 & 32330 & 435690 & 7.4 \\
\hline Valencia & 95477 & 18138 & 77339 & 604544 & 12.8 \\
\hline Alicante & 53907 & 7519 & 46388 & 256339 & 18.1 \\
\hline TOTAL & 185098 & 29041 & 156057 & 1296573 & 12.0 \\
\hline
\end{tabular}

Tal como se observa, el esfuerzo repoblador presenta notables diferencias entre provincias. Aunque es en la provincia de Valencia en la que más se ha repoblado, en proporción es en la provincia de Alicante donde mayor esfuerzo repoblador se ha realizado ya que se ha trabajado casi sobre una de cada cinco hectáreas forestales. Por otro lado, mientras que en Valencia el porcentaje de superficie forestal es aproximadamente la mitad que el autonómico y este porcentaje se mantiene respecto a la distribución de repoblaciones, no ocurre igual en el resto de provincias.

En Castellón se concentran menos del 20\% de las superficies repobladas, mientras que supone más del $30 \%$ de la superficie forestal autonómica. Contrariamente sucede en Alicante, donde se concentra menos del $20 \%$ de la superficie forestal pero casi el $30 \%$ de las repoblaciones.

El menor desarrollo de este tipo de actuaciones en Castellón puede estar asociado tanto a una menor existencia de terrenos forestales gestionados por la administración, especialmente si se compara con Valencia, así como a una menor limitación climática que dificulte la evolución y recuperación de la vegetación, especialmente si se compara con Alicante, existiendo menor necesidad de ejecutar estos trabajos de restauración.

Las superposiciones, es decir, las zonas en las que se ha repoblado en más de una ocasión, supone cerca de un 16\% del total, destacando que en Valencia 1 de cada 5 hectáreas repobladas se ha repoblado en más de una ocasión, lo que puede ser debido al poco éxito de la primera actuación o a la afección por el fuego de esas zonas que requieren de nuevo la intervención del hombre para la recuperación de la cubierta forestal.

En Castellón menos del 10\% de la superficie repoblada se ha realizado en más de una ocasión y en Alicante cerca del 14\%.

\subsection{Evolución histórica de la actividad repobladora}

La máxima actividad repobladora se registra a mitad del siglo XX, coincidiendo con el auge en la ejecución del Plan Nacional de Repoblaciones, siendo el año 1957 el año en que se repobló más superficie en España, con 143968 ha (Pemán y Vadell, 2009). Esto coincide con que el periodo 1950 y 1959 fuera el de máxima actividad 


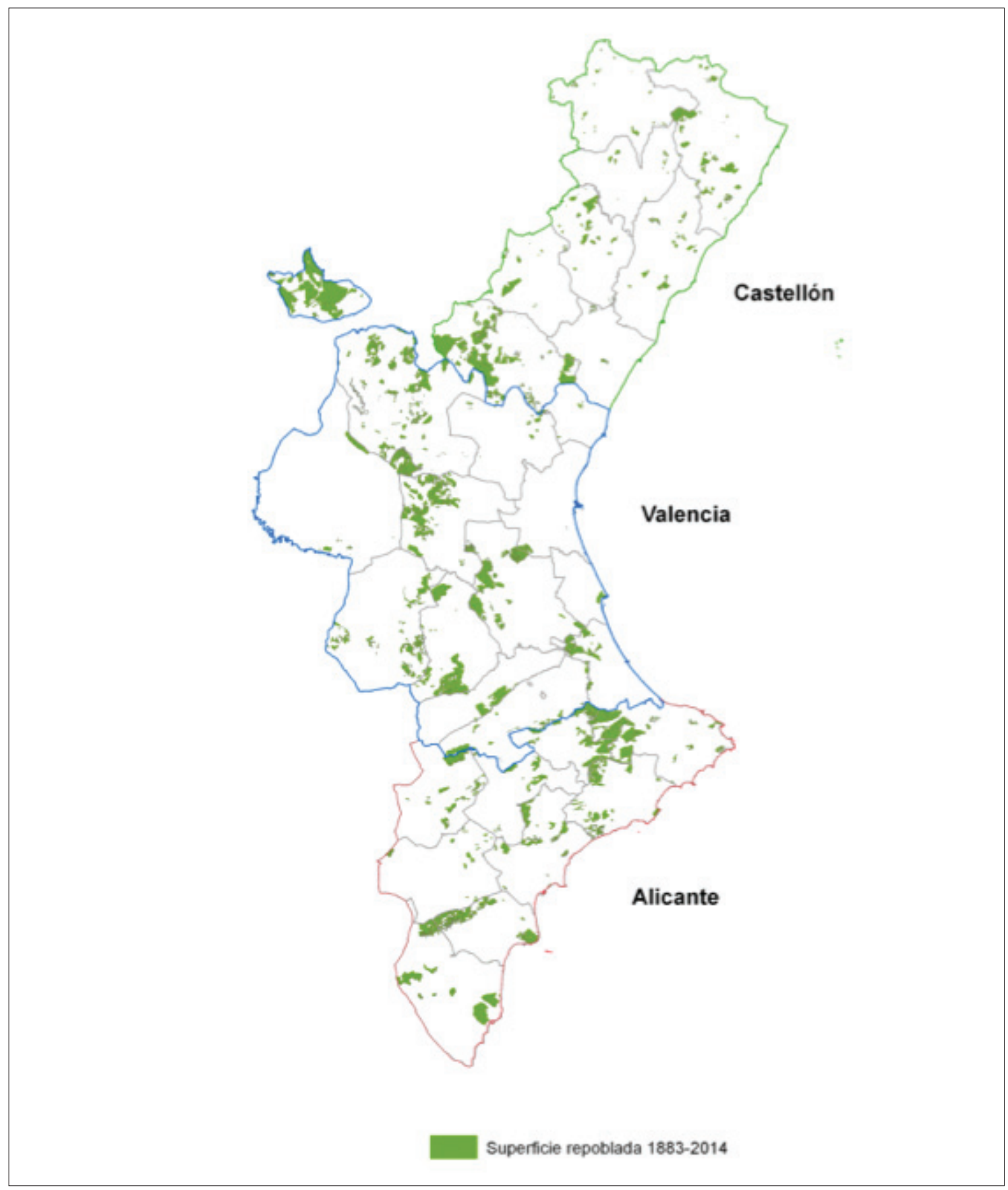

Figura 1. Distribución superficial de las repoblaciones ejecutadas por las administraciones públicas en la Comunitat Valenciana en el periodo 1883-2014.

repobladora también en la Comunitat Valenciana (Figura 2), ejecutándose 50576 ha de repoblación (más de 5000 anuales).

Las épocas en las que se ha registrado menor actividad comprenden el período hasta 1930 y el de los años 2010-2014, en el que se han repoblado menos de 300 ha al año. 


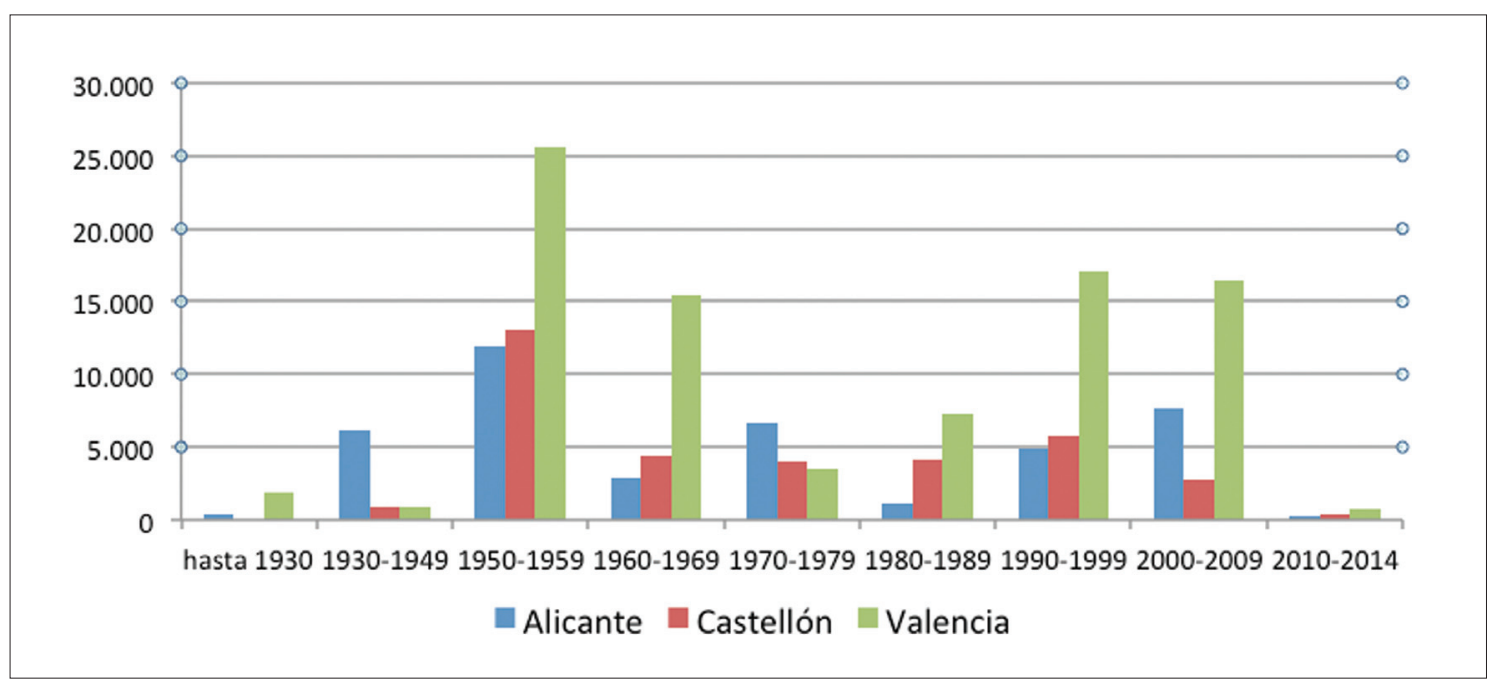

Figura 2. Evolución de la actividad repobladora en el periodo 1883-2014 por provincias y por décadas sin eliminar áreas repobladas más de una vez (superposiciones).

A finales del siglo XIX y principios del XX, la actividad repobladora no fue de gran importancia (Tabla 2), aunque sí se establecieron las bases para las importantes actuaciones que se desarrollaron posteriormente (Pemán y Navarro, 1998). De hecho en Castellón no se recoge la existencia de ningún trabajo de este tipo y en Alicante y Valencia puede considerarse anecdóticos, con una media de 40 hectáreas anuales. Este resultado destaca que algunos proyectos antiguos no han sido recogidos por la cartografía como por ejemplo la repoblación de las dunas de Guardamar del Segura, que fueron ejecutadas a principios del siglo XX y ocuparon más de 700 ha.

El segundo periodo analizado (1940-1975) coincide, tal como se ha ido comentando, con la época de mayor actividad repobladora, en la que se ejecutó más de la mitad de la superficie repoblada en todo el periodo analizado, superando las 2.600 ha repobladas al año. Durante todo este periodo la intensidad no fue igual, ya que creció hasta la década de los 50, donde alcanzó el máximo, para posteriormente ir descendiendo.

Tabla 2. Superficie total y media repoblada por períodos históricos y provincia (excluyendo las de fecha desconocida).

\begin{tabular}{|c|c|c|c|c|c|}
\hline Periodo & Alicante & Castellón & Valencia & Total & Media anual \\
\hline $1883-1939$ & 395 & 0 & 1884 & 2279 & 40 \\
\hline $1940-1975$ & 26413 & 22180 & 45332 & 93925 & 2609 \\
\hline $1976-1984$ & 2281 & 2166 & 4654 & 9100 & 1011 \\
\hline $1985-2014$ & 12636 & 11171 & 36898 & 60706 & 2024 \\
\hline TOTAL & 41725 & 35517 & 86884 & 166010 & 1258 \\
\hline
\end{tabular}

Este descenso continuó en el periodo comprendido entre 1976 y 1984, donde apenas se superaron las 1000 ha anuales. Finalmente, tras la cesión de las competen- 
cias a las autonomías, la actividad repobladora volvió a recuperarse superando las 2000 ha al año (Tabla 2), aunque sin alcanzar los niveles de mitad de siglo, a pesar de que hubo un interés político por restaurar las grandes zonas afectadas por las grandes incendios, nunca llegó a materializarse en su totalidad (Pérez, 2005)

\subsection{Efectividad de la actividad repobladora}

Al analizar el porcentaje de la superficie repoblada anteriormente a 1996 catalogada como arbolada en el IFN3, se observa que únicamente la mitad de la superficie repoblada presenta en 2006 una masa arbolada. Si se hubieran considerado los grandes incendios de 2012, es probable que este valor aún se redujera más (Tabla 3).

En este caso existen importantes diferencias entre provincias, presentando Castellón un grado de éxito muy elevado, en donde el $70 \%$ de la superficie repoblada alcanza masas arboladas, mientras que este porcentaje se reduce drásticamente en la provincia de Alicante con poco más del $40 \%$.

Tabla 3. Superficie repoblada (ha) hasta 1996, con y sin superposiciones y su efectividad..

\begin{tabular}{|c|c|c|c|c|}
\hline Provincia & Total & Sin superposiciones & Sup. rep. arbolada en IFN3 & Efectividad \\
\hline Castellón & 31077 & 29841 & 20956 & 70.2 \\
\hline Valencia & 73658 & 65256 & 30688 & 47.0 \\
\hline Alicante & 44838 & 42206 & 16980 & 40.2 \\
\hline Total CV & 149573 & 137303 & 68625 & 50.0 \\
\hline
\end{tabular}

\subsection{Superficie repoblada que ha sufrido posteriormente un incendio}

La principal explicación de que una zona repoblada no llegue a formar una masa arbolada, considerando que tanto el diseño como la ejecución de ésta son adecuados, es la afección por parte de un incendio forestal.

Tabla 4. Superficie repoblada (sin superposiciones) hasta 1996 y su afección por los incendios forestales en el periodo 1978-2006 para Valencia y 1993-2006 para Castellón y Alicante.

\begin{tabular}{|c|c|c|c|c|c|}
\hline & & \multicolumn{4}{|c|}{ Incendiada 1978-2006 } \\
\hline Provincia & $\begin{array}{c}\text { Total } \\
\text { (Sin superposiciones) }\end{array}$ & Total & $\begin{array}{c}\text { Arbolada } \\
\text { en IFN3 }\end{array}$ & $\begin{array}{c}\text { No arbolada } \\
\text { en IFN3 }\end{array}$ & $\begin{array}{c}\text { \% sup. repoblada } \\
\text { no arbolada } \\
\text { por incendio }\end{array}$ \\
\hline Castellón & 29841 & 641 & 229 & 412 & 1.4 \\
\hline Valencia & 65256 & 36567 & 11245 & 25322 & 38.8 \\
\hline Alicante & 42206 & 374 & 48 & 326 & 0.8 \\
\hline Total CV & 137303 & 37582 & 11522 & 26060 & 19.0 \\
\hline
\end{tabular}


Del total de superficie repoblada en la comunidad autónoma entre 1883 y 1996, cerca de un 30\% (Tab. 4) ha sufrido por lo menos un incendio desde 1978 a 2006 (en la provincia de Valencia, desde 1993 en Castellón y Alicante).

Sin embargo, esta problemática viene marcada por grandes diferencias entre provincias, ya que mientras que en Valencia más del 56\% de la superficie repoblada se ha visto afectada por un incendio, en Castellón y Alicante este porcentaje se reduce por debajo del 3\%. En estos valores evidentemente influye el hecho de que se dispone de mayor información cartográfica de incendios forestales que en las otras provincias (desde 1978 no desde 1993).

Dentro de la superficie repoblada y afectada por el fuego, la mayoría de ella (en torno a un 70\%) aparece como no arbolada en el IFN3, siendo éste un resultado esperado.

Las zonas repobladas afectadas por un incendio, que mantienen su masa arbolada, pueden deberse a que ha discurrido el suficiente tiempo para su regeneración tras el incendio, o a que el incendio no alcanzó las copas y permitió la supervivencia del arbolado.

Hay que recordar que el IFN3 considera un monte como arbolado cuando la FCC arbórea es superior al 5\%.

\section{Conclusiones}

Las repoblaciones forestales son el método más usual para la recuperación de la cubierta vegetal.

El esfuerzo repoblador realizado en la Comunitat Valenciana ha sido similar al del resto de comunidades autónomas. Únicamente la mitad de las repoblaciones anteriores a 1996 forman masas arboladas, en gran parte debido a los incendios forestales que las han asolado, principalmente en la provincia de Valencia, lo que evidencia la necesidad del mantenimiento y gestión en el tiempo de las actuaciones que se realicen.

La información cartográfica disponible, a pesar de tener sus limitaciones, es adecuada para obtener una imagen de la situación actual de las repoblaciones ejecutadas en nuestro ámbito.

Sería adecuado mantener y actualizar estas cartografías así como continuar con estudios que analicen la relación entre la recurrencia de incendios forestales y la ejecución de repoblaciones

El esclarecimiento de las relaciones entre repoblaciones e incendios requiere de un análisis de mayor profundidad que incida en el tiempo transcurrido entre los trabajos de repoblación y el paso de un incendio y la recurrencia de incendios sobre un mismo territorio.

Considerar el riesgo de incendio a la hora de planificar repoblaciones podría servir para optimizar las inversiones realizadas. En cualquier caso, es evidente la necesidad del mantenimiento y gestión en el tiempo de las actuaciones que se realicen, para evitar su pérdida. 


\section{Agradecimientos}

Al Servicio de Ordenación y Gestión Forestal de la Conselleria d'Infraestructures Territori i Medi Ambient y al Servicio de Prevención y Extinción de la Conselleria de Governació i Justícia, por las facilidades ofrecidas en el acceso a la información numérica y cartográfica necesaria para llevar a cabo este trabajo.

\section{Bibliografía}

Costa P. et al 2011. La Prevención de los Grandes Incendios Forestales adaptada al Incendio Tipo. GRAF. Generalitat de Catalunya.

De Las Heras, J. et al. 2011. Restauración y manejo de pinares de pino carrasco tras incendio en el sureste de la península ibérica. Boletín del CIDEU 10: 63-79

Inventario Forestal Nacional III (IFN3), 2008. Tercer inventario forestal nacional en las provincias de Alicante, Castellón y Valencia. Madrid: Ministerio de Medio Ambiente, Medio Rural y Marino de España. No publicado

Mac Donald, L.H.; Robichaud, P. R., 2008. Post-fire erosion and the effectiveness of emergency rehabilitation treatments over time. Stream Notes. Stream Systems Technology Center. USDA Forest Service. January 2008.

Martínez Hermosilla P., 1953. La repoblación forestal en España y su importancia para la agricultura y la economía pública. Revista de Estudios Agrosociales, ISSN 0034-8155, $\mathrm{N}^{\circ} .5,1953$, págs. 37-48.

Montero G., Grau, J.M., Ruiz-Peinado R., Ortega C. y Cañellas L., 2000. Tablas de producción para Pinus halepensis MilI. Dpto. de Selvicultura CIFOR-INIA. Cuad. Soc Esp. Cien. For. 10: 183-188

Pemán J. y Navarro R., 1998. Repoblaciones forestales. Universitat de Lleida.

Pemán J y Vadell E., 2009. Reconstrucción de la estadística de la actividad repobladora desde 1879 hasta nuestros días. $5^{\circ}$ Congreso Forestal Español

Reyna Doménech, S. y Fernández Guijarro., 2000. Evolución histórica de la masas forestales en la Comunidad Valenciana del S.XVIII a la actualidad, la información de Cavanilles y Delacroix. Ponencia en Mesa Redonda Sobre los montes valencianos ayer. Sociedad Económica de Amigos del País

PATFOR, 2013. Plan de Acción Territorial Forestal de la Comunitat Valenciana. GVA.

Pérez Baldó F. 2005.La repoblación forestal en la Comunidad Valenciana (aplicación en el Rincón de Ademuz). IV Ciclo sobre los Montes Valencianos. Real Sociedad Económica de Amigos del País.

Serrada, R. 2000. Apuntes de Repoblaciones Forestales. FUCOVASA. Madrid.

Vélez, R. 2000. La defensa contra incendios forestales. Fundamentos y experiencias. McGraw hill, Madrid 
